\title{
Segmentation and Evaluation of COVID-19 Lesion from CT scan Slices - A Study with Kapur/Otsu Function and Cuckoo Search Algorithm
}

\section{Suresh Chandra Satapathy}

Kalinga Institute of Industrial Technology

D. Jude Hemanth

Karunya Institute of Technology and Sciences

Seifedine Kadry

Beirut Arab University

Gunasekaran Manogaran

University of California, Asia University

Naeem M S Hannon

Universiti Teknologi MARA

V. Rajinikanth ( $\nabla$ v.rajinikanth@ieee.org )

St. Joseph's College of Engineering

\section{Research Article}

Keywords: COVID-19 infection, pneumonia, Kapur/Otsu thresholding, cuckoo search algorithm, validation

Posted Date: July 12th, 2020

DOI: https://doi.org/10.21203/rs.3.rs-40148/v1

License: (1) This work is licensed under a Creative Commons Attribution 4.0 International License.

Read Full License 


\title{
Segmentation and Evaluation of COVID-19 Lesion from CT scan Slices - A Study with Kapur/Otsu Function and Cuckoo Search Algorithm
}

\author{
Suresh Chandra Satapathy ${ }^{1}$, D. Jude Hemanth ${ }^{2}$, Seifedine Kadry ${ }^{3}$, Gunasekaran Manogaran ${ }^{4,5}$, \\ Naeem M S Hannon ${ }^{6}$, V. Rajinikanth ${ }^{7, *}$ \\ ${ }^{1}$ School of Computer Engineering, Kalinga Institute of Industrial Technology (Deemed to Be University), Bhubaneswar, 751024, \\ India \\ ${ }^{2}$ Department of ECE, Karunya Institute of Technology and Sciences, Coimbatore, 641 114, India \\ ${ }^{3}$ Department of Mathematics and Computer Science, Faculty of Science, Beirut Arab University, Beirut, 11072809, Lebanon \\ ${ }^{4}$ University of California, Davis, CA 95616, USA \\ ${ }^{5}$ Computer Science and Information Engineering, Asia University, Lioufeng Rd., Wufeng, Taichung 41354, Taiwan \\ ${ }^{6}$ Faculty of Electrical Engineering, Universiti Teknologi MARA, Malaysia \\ ${ }^{7}$ Department of Electronics and Instrumentation Engineering, St. Joseph's College of Engineering, Chennai, 600 119, India \\ ${ }^{*}$ Corresponding Author: V. Rajinikanth, Email: v.rajinikanth@ieee.org
}

\begin{abstract}
Infection/disease in lung is one of the acute illnesses in humans. Pneumonia is one of the major lung diseases and each year; the death rate due to the untreated pneumonia is on rise globally. From December 2019; the pneumonia caused by the Coronavirus Disease (COVID-19) has emerged as a global threat due to its rapidity. The clinical level assessment of the COVID-19 is normally performed with the Computed-Tomography scan Slice (CTS) or the Chest X-ray. This research aims to propose an image processing system to examine the COVID-19 infection in CTS. This work implements CuckooSearch-Algorithm (CSA) monitored Kapur/Otsu image thresholding and a chosen image segmentation procedure to extract the pneumonia infection. After extracting the COVID-19 infection from the CTS, a relative assessment is then executed with the Ground-Truth-Image (GTI) offered by a radiologist and the essential performance measures are then computed to confirm the superiority of the proposed technique. This work also presents a comparative assessment among the segmentation procedures, such as Level-Set (LS) and Chan-Vese (CV) methods. The experimental outcome authenticates that, the results by Kapur and Otsu threshold are approximately similar when the LS is implemented and the CV with the Otsu presents better values of Jaccard, Dice and Accuracy compared to other methods presented in this research.
\end{abstract}

Keywords: COVID-19 infection; pneumonia; Kapur/Otsu thresholding; cuckoo search algorithm; validation

\section{Introduction}

In the current era, the advancement in science and engineering aids the humans to have a better living ambiance with greater facilities. Even though the medical facilities are improved to provide the appropriate treatment process; the disease rate in humans are rising due to their lifestyle and the change in the environmental conditions. In humans, the disease in lung arises due to various infections and this abnormality will severely affect the entire physiological system in human. Pneumonia is one of the major lung abnormalities in infants (age $<5$ years) and elderly people (age $>65$ years) and the untreated pneumonia will lead to death. The pneumonia is a kind of infectious disease caused by microorganisms, 
such as bacteria, fungi and viruses. In 2017 the World-Health-Organization (WHO) reports that, the pneumonia killed 8, 08, 694 children (age $<5$ years) worldwide [1].

The premature detection of pneumonia and the possible treatment implementation will reduce the death rate in humans [2,3]. The lung infection due to pneumonia is normally assessed using the imaging procedures, such as the Computed-Tomography (CT) and Chest Radiographs (X-ray). In these approaches, the patient is screened using a chosen imaging method and after the screening process, the doctor will examine the chest images and based on his observation, a possible treatment procedure is implemented. Due to its significance, a number of pneumonia detection procedures are already proposed and implemented by the researchers [4-6].

The pneumonia due to the Coronavirus Disease (COVID-19) is one of the novel diseases which affected a large human group worldwide [7,8]. This disease was first discovered in Wuhan, China in December 2019 [9,10] and due to its outbreak, the infection rate is gradually increasing. The earlier research works on COVID-19 confirms that, COVID-19 affects the respiratory tract and causes the severe pneumonia and the premature detection will help to cure the patients [11-13]. The common procedures followed in the clinical level detection of the COVID-19 include; (i) Reverse Transcription-Polymerase Chain Reaction (RT-PCR) test and (ii) Image (CT scan /Chest X-ray) assisted detection. The RT-PCR is a laboratory test initially implemented to test the collected sample from the COVID-19 patient. If the RTPCR test result is positive, then the doctor will suggest for the image assisted detection procedure to discover the infection and its severity. The CT scan/Chest X-ray is recorded using the radiology facility and the recorded images are then directed to the doctor for further assessment. The outcome of the image assisted detection is then considered to plan for the appropriate treatment to recover the patient from COVID-19 infection.

Due to its three-dimensional view and good visibility, the CT scan Slices (CTS) are widely used by the doctors to detect the COVID-19 disease compared to the Chest X-ray [14]. Due to its infection rate and harshness, the COVID-19 infected patients are kept in isolated and controlled surroundings and when the infection rate increases, the patient count also increases; which will increase the burden of the doctors and the pulmonologist. To reduce the diagnostic burden; recently a number of automated pneumonia detection system using CTS/Chest X-ray was proposed by the researchers $[15,16]$. The existing methods include; (i) Segmentation techniques, (ii) Machine-Learning-Schemes (MLS) and (iii) Deep-LearningSystems (DLS) [11,12]. The existing methods are implemented on a class of real time and benchmark images and validated using a chosen software platforms The earlier works in the literature also confirms that, the assessment of the COVID-19 disease using a chosen image processing scheme substantially reduces the burden and helps to implement the treatment implementation process [13-16].

The aim of the proposed research is to develop an image evaluation scheme using the hybrid methods existing in the literature [17-20]. The Hybrid-Image-Processing-System (HIPS) can be developed by integrating the chosen multi-threshold scheme with a chosen segmentation technique. The Performance of the multi-thresholding can be monitored and improved by employing an appropriate heuristic algorithm. The proposed research has the following steps; (i) Collection of CTS from the benchmark dataset, (ii) Artifact removal using threshold-filter, (iii) Tri-level thresholding with CuckooSearch-Algorithm (CSA) and Kapur/Otsu function, (iv) Extracting the Region-of-Interest (ROI) using Level-Set (LS) or Chan-Vese (CV) segmentation, and (v) Comparison of the ROI with the Ground-TruthImage (GTI) and computation of performance measures.

In this work, the COVID-19 dataset provided by Jun et al. [21] is considered for the assessment. From this dataset, 100 numbers of images ( 10 patients $\times 10$ image $/$ patient $=100$ images $)$ of dimension 630x630x1pixels are considered for the evaluation. In order to improve the search efficiency of the CSA, a chaotic operator known as Lorenz-Attractor [22,23] is implemented and the considered test images are separately processed with Kapur+LS, Kapur+CV, Otsu+LS and Otsu+CV. Finally, the performance of proposed HIPS is confirmed using the mean values of Jaccard, Dice and segmentation accuracy attained using an experimental study implemented with MATLAB software. 
This research is presented as follows; section 2 discusses the methodology, sections 3 and 4 presents the experimental outcome on a chosen COVID-19 dataset and the conclusion respectively.

\section{Context}

Due to its medical emergency and its medical emergence, recently, a considerable number of procedures are proposed to analyze the COVID-19 infection in humans using imaging modalities, such as CT scan and Chest X-ray and based on the attained outcome; the essential treatment procedures are implemented to recover the patient from the infection. After confirming the infection using the prescribed clinical test (RT-PCR), an image assisted assessment is then employed as discussed in earlier works $[11,12]$.

In the recent literature, a considerable number of image assisted evaluation of the COVID-19 is presented using the clinical grade and benchmark images and the result of these procedures confirmed that, a carefully designed image examination procedure will help to identify the COVID-19 infection and its harshness; which plays a vital role during the treatment planning and implementation process. Further, the choice of the medical aids, such as the medicine, appropriate dosage level, and the need for the ventilation unit will be decided based on the decision reached with the help of the result of the image assessment task.

A summary of the recently proposed image examination procedures on the COVID-19 infection is presented in Table 1.

\begin{tabular}{|c|c|c|}
\hline Reference & $\begin{array}{l}\text { Image } \\
\text { modality }\end{array}$ & Implemented methodology and outcome \\
\hline Ozturk et al. [41] & Chest X-Ray & $\begin{array}{l}\text { Deep neural network based assessment of } \\
\text { COVID- } 19 \text { lesion using a five-fold cross } \\
\text { validation. This method helped to attain the } \\
\text { following result: } \\
\text { Accuracy }=98.08 \% \text {, Precision }=98.03 \% \text {, } \\
\text { Sensitivity }=95.13 \% \text {, Specificity }=95.3 \% \text { and } \mathrm{F}- \\
\text { score }=96.52 \%\end{array}$ \\
\hline Alazab et al. [42] & Chest X-Ray & $\begin{array}{l}\text { This work implements the detection of COVID-19 } \\
\text { infection using with and without data } \\
\text { augmentation and achieved a classification } \\
\text { accuracy of } 99 \% \text {. }\end{array}$ \\
\hline Singh et al. [43] & CT scan slice & $\begin{array}{l}\text { Binary classification of COVID-19 lesion is } \\
\text { implemented using multi-objective differential } \\
\text { evolution-based convolutional neural networks } \\
\text { and this research reported a better performance } \\
\text { value. }\end{array}$ \\
\hline Ardakani et al. [44] & CT scan slice & $\begin{array}{l}\text { This work presents a detailed analysis of ten well } \\
\text { known deep-learning architectures and attained a } \\
\text { maximum classification accuracy of } 86.27 \% \text {. }\end{array}$ \\
\hline Ahuja et al. [45] & CT scan slice & $\begin{array}{l}\text { Assesment of the COVID-19 infection is } \\
\text { implemented using deep transfer learning } \\
\text { technique and the ResNet18 architecture helped to } \\
\text { attain a better classification accuracy compared to } \\
\text { other systems considered. }\end{array}$ \\
\hline Rajinikanth et al. [11] & CT scan slice & $\begin{array}{l}\text { The segmentation of COVID-19 lesion using the } \\
\text { Harmony-search and Otsu's thresholding and }\end{array}$ \\
\hline
\end{tabular}




\begin{tabular}{|c|c|c|}
\hline & & $\begin{array}{l}\text { water shed segmentation is implemented and a } \\
\text { disease infection rate is computed based on the } \\
\text { pixel ratio between the infected section and lung } \\
\text { section. }\end{array}$ \\
\hline Rajinikanth et al. [12] & CT scan slice & $\begin{array}{l}\text { This work presented an automated segmentation } \\
\text { procedure based on the firefly algorithm and } \\
\text { Shannon's entropy based thresholding and } \\
\text { Markov-Random-Field based segmentation. }\end{array}$ \\
\hline Kadry et al. [13] & CT scan slice & $\begin{array}{l}\text { This system developed a machine-learning system } \\
\text { to classify the lung CT scan slices into COVID-19 } \\
\text { and non-COVID class and this work helped to } \\
\text { attain a classification accuracy of } 89.80 \% \text {. }\end{array}$ \\
\hline Dey et al. [14] & CT scan slice & $\begin{array}{l}\text { This work implemented an integration of } \\
\text { segmentation and classification task using social } \\
\text { group optimization based Kapur's thresholding } \\
\text { and morphological segmentation. This work } \\
\text { presented a overall segmentation accuracy of } \\
>91 \% \text { and classification accuracy of } 87 \% \text { on the } \\
\text { considered COVID-19 database. }\end{array}$ \\
\hline
\end{tabular}

The results existing in Table 1 confirms that, image assisted detection plays a vital role in detecting the COVID-19 infection with better accuracy. Further, this system may considerably reduce the diagnostic burden of the pulmonologist; by providing the essential information regarding the patient; who admitted with COVID-19 infection. If an appropriately designed/tuned image processing system is available, then the detection of the infection rate will be accurate.

The earlier works confirms that, a number of segmentation, machine learning and deep learning techniques are proposed by the researchers to evaluate and detect the COVID-19 infection with better accuracy. Even though the experimental results are good, every method has its limitations, such as initial tuning, implementation and validation, etc. Further, the detection time also plays a vital role in clinical level diagnosis.

The proposed research work aims to implement a simple and efficient semi-automated image processing scheme to extract the COVID-19 lesion from the lung CTS with better accuracy for further assessment. This system is developed by integrating the image pre-processing and post-processing system and its performance if validated by considering the benchmark COVID-19 dataset existing at [21]. The experimental outcome of the proposed system confirms that, the results attained with proposed system are clinically significant.

\section{Materials and Methods}

Accurate examination COVID-19 lesion is very essential for faster diagnosis and treatment implementation. Further, the procedures to be implemented should be user friendly and easily reproducible. The ultimate aim of the image processing system for the diagnosis of the COVID-19 should be simple and accurate. This work proposes HIPS to examine the CTS of the COVID-19 patient with better accuracy. The various procedures involved in this system are clearly depicted in Figure 1.

The various sections of the HIPS are; (i) Data collection and initial processing, (ii) Artifact removal for better detection, (iii) Pre-processing to enhance the pixel groups of the COVID-19 lesion, (iv) Implementation of a chosen segmentation technique to extract the lesion, (v) Comparison and validation of the proposed method with a reference image given by a disease expert. 


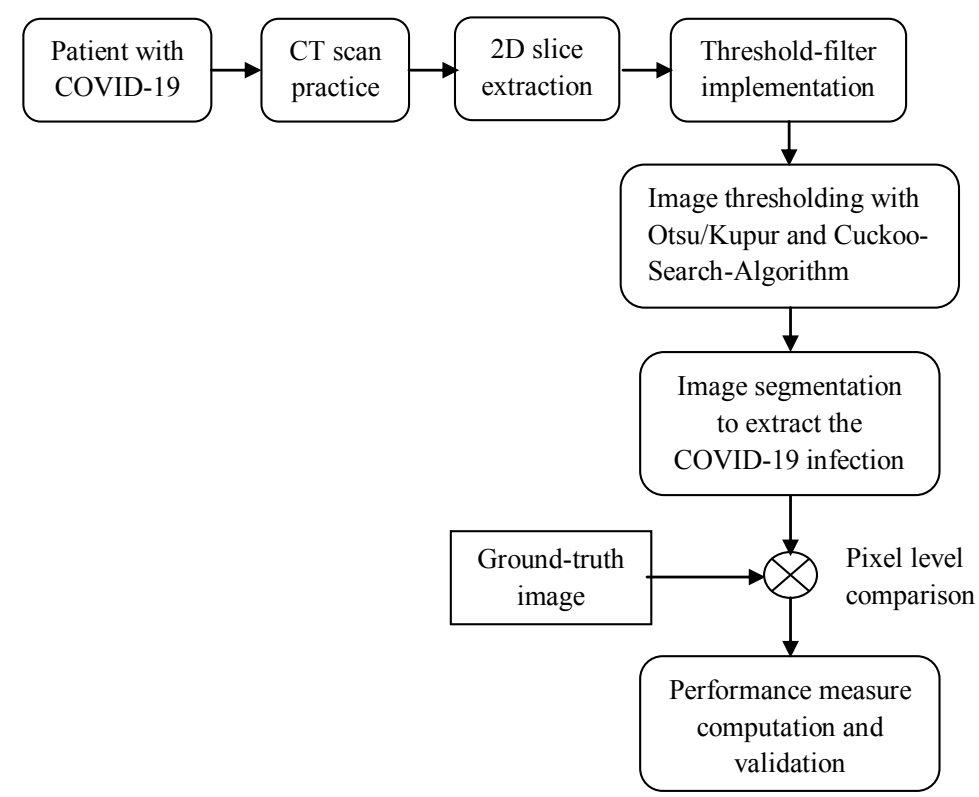

Figure 1: Various stages involved in the proposed image processing scheme

When the RT-PCR test result of a patient is positive; then a recommended radiology assisted image screening is implemented to record the respiratory tract for further examination. The CTS is the widely preferred imaging modality, which offers a 3D image of the lung. Examination of the 3D lung section with computerized technique is quite complex and hence, a 2D slice with axial-view is considered in this work. The 3D to 2D conversion is executed using the ITK-snap tool $[39,40]$; which offered an image of dimension 630x630x1 pixels. Later, a threshold filter discussed in [11] is employed to separate the 2D lung CTS into the artifact and the Section-of-Interest (SOI). The SOI is then enhanced using a chosen thresholding procedure (Kapur/Otsu) monitored by the chaotic CSA. The COVID-19 infection (ROI) from the threshold image is then extracted using the LS/CV technique. The extracted ROI is compared against the GTI existing in the image database and this comparison helps to achieve essential image performance measures. Based on the segmentation accuracy, the performance of the proposed HIPS is validated.

\subsection{Image Thresholding}

Image thresholding is a widely adopted technique to process a considerable number of grayscale avs RGB scale images and recently, it adopted to enhance a class of medical images. In the proposed work, after extracting the 2D CTS from the COVID-19 dataset, the normal lung section and the other image parts are eliminated with the help of a threshold-filter. The threshold filter separates the image into two sections and helps to provide the image SOI to be evaluated further. The SOI is then processed with the HIPS and finally the infection due to the COVID-19 is extracted and evaluated. The tri-level threshold is initially implemented in the HIPS to enhance the image using the chaotic CSA and Kapur/Otsu function. This process helps to enhance the test picture by grouping the image pixels into background, normal section and COVID-19 infection.

\subsection{Cuckoo search algorithm}

The traditional CSA is proposed by Yang and Deb in 2009 [24,25] and over a decade, it is widely used to solve a variety of image processing applications [26-28]. In the literature, a considerable number of enhancement procedures are proposed to improve the optimization search of the CSA and in this work, Lorenz-Attractor based chaotic search is implemented to enhance the search ability of the CSA. 
The traditional CSA is developed by mimicking the breeding artifice followed by parasitical cuckoos [24]. The CSA is developed by considering the following assumptions; (i) every cuckoo deposit an egg in a randomly selected nest of the host birds, (ii) the nest with eminent enduring egg will be carried to the subsequent invention, and (iii) For a chosen threshold problem, the amount of host-bird's nest is fixed and it may identify the egg of cuckoo with a probability $P_{a} \in[0,1]$. When the host identifies the egg, it may eliminate the egg or abandon the current nest and the host wild build a new nest.

Above said assumptions are accounted to create the mathematical model of the CSA. The implementation steps in CSA are quite simple compared to other existing approaches and the proposed CSA is depicted in Eqn. (1).

$$
X_{i}^{n+1}=X_{i}^{n}+\sigma \oplus L A
$$

where, $X_{i}^{n}=$ early location, $X_{i}^{n+l}=$ updated location, $\sigma=$ step size (chosen as 1.2), $\oplus=$ entry wise multiplication, and $=L A=$ Lorenz-Attractor search $[22,23]$.

The pseudo-code of the Chaotic CSA (CCSA) is depicted below:

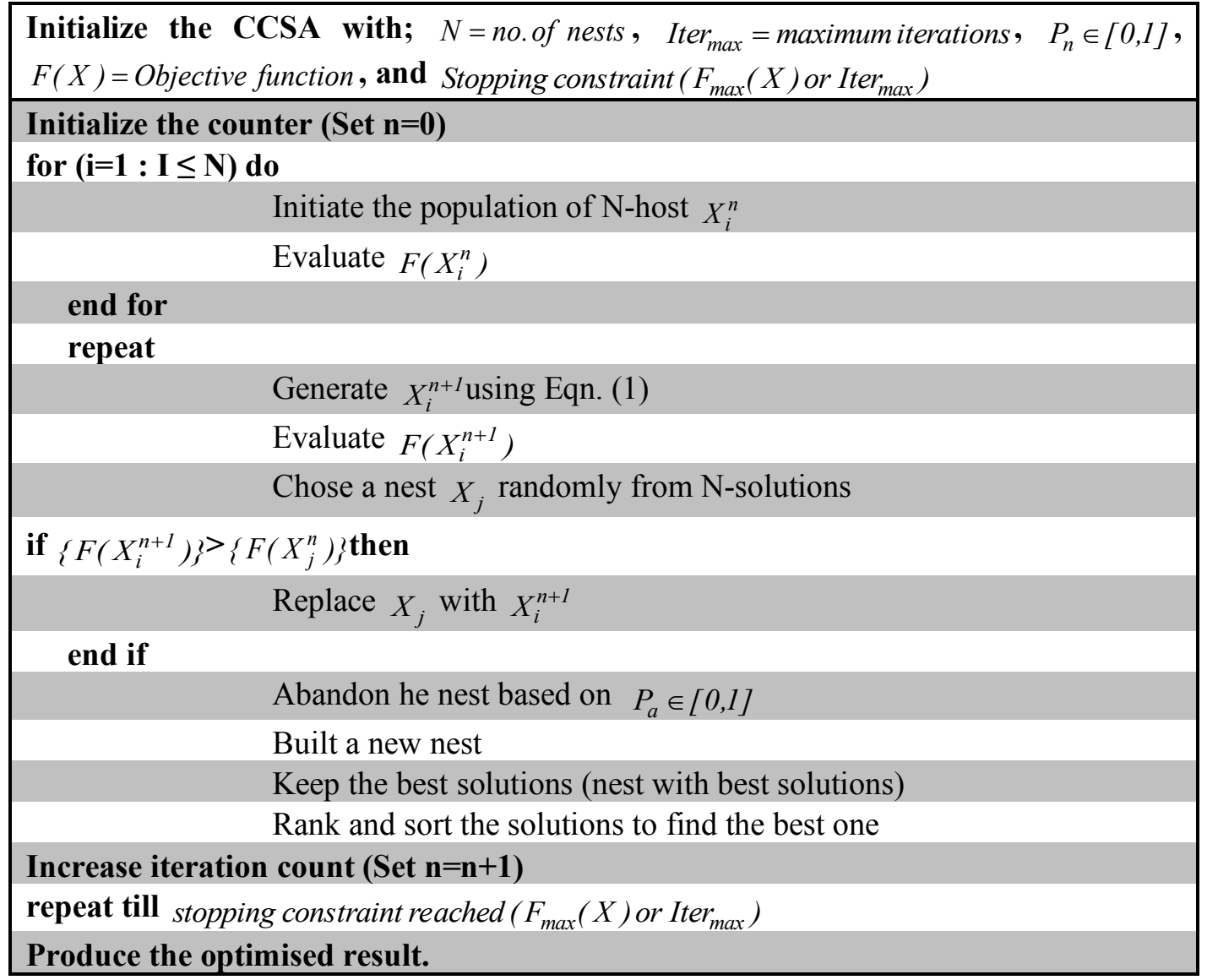

\subsection{Kapur's function}

Entropy assisted image threshold is widely adopted in the literature to pre-process the test images using a chosen threshold $(T h)$ value. Kapur's entropy $(\mathrm{KE})$ is one of the proven methods, widely adopted to examine a class of medical information ranging from bio-signals to bio-images $[29,30]$.

The KE implemented in this work is as follows;

Let , the chosen image has $T h$ number of threshold ranging as; $T h=\left(t_{h 1}, t_{h 2}, \ldots, t_{h L-1}\right)$ for $\mathrm{L}=256$. 
Let, the chosen image has a total pixel size of $S$; then the pixel distribution in the image with respect to its frequency distribution $s(d)$ can be denoted as;

$$
S=s(0)+s(1)+, \ldots+s(L-1)
$$

Then the probability of $d^{\text {th }}$ intensity-level is denoted by;

$p(d)=s(d) / S$

The threshold process can be used to divide the image pixels into $T+1$ clusters and then the KE for every cluster is calculated individually and pooled to get the ultimate entropy.

For a tri-level threshold problem, the computed entropy will be;

$$
\begin{aligned}
& S\left(t_{h 1}, t_{h 2}, t_{h 3}\right)=e_{0}+e_{1}+e_{2} \\
& e_{0}=-\sum_{d=0}^{d=t_{h 1}-1} \frac{p_{d}}{\omega_{0}} \ln \frac{p_{d}}{\omega_{0}}, \omega_{0}=\sum_{d=0}^{d=t_{h 1}-1} p_{d} \\
& e_{1}=-\sum_{d=t_{h 1}-1}^{d=t_{h 2}-1} \frac{p_{d}}{\omega_{1}} \ln \frac{p_{d}}{\omega_{1}}, \omega_{1}=\sum_{d=t_{h 1}-1}^{d=t_{h 2}-1} p_{d} \\
& e_{2}=-\sum_{d=t_{h 2}-1}^{d=t_{h 3}-1} \frac{p_{d}}{\omega_{2}} \ln \frac{p_{d}}{\omega_{2}}, \omega_{2}=\sum_{d=t_{h 2}-1}^{d=t_{h 3}-1} p_{d}
\end{aligned}
$$

where $e=$ entropy, $p=$ probability distribution, and $\omega=$ probability occurrence.

During this operation, the objective is to find; $K E_{\max }=F_{K E_{\max }}(T h)$

\subsection{Otsu's function}

Otsu's Between-Class-Variance (OBCV) assisted thresholding is considered in a number of earlier works to pre-process the clinical grade images [11]. The mathematical description of this process can be found in [31,32].

Let for a given image, the thresholds are $T h=\left(t_{h 1}, t_{h 2}, \ldots, t_{h L-1}\right)$ for $\mathrm{L}=256$. The probability distribution of the images is; $B_{0}, B_{1}$ and $B_{2}$ with a varied thresholds;

$$
B_{0}=\frac{p_{0}}{\eta_{0}(T h)} \ldots \frac{p_{t_{h 1}-1}}{\eta_{0}(T h)}, B_{1}=\frac{p_{t_{h 1}}}{\eta_{1}(T h)} \ldots \frac{p_{t_{h 2}-1}}{\eta_{1}(T h)} \text { and } B_{2}=\frac{p_{t_{h 2}}}{\eta_{2}(T h)} \ldots \frac{p_{L-1}}{\eta_{2}(T h)}
$$

where $\eta_{0}(T h)=\sum_{d=0}^{d=t_{h 1}-1} p_{d}, \eta_{1}(T h)=\sum_{d=t_{h 1}-1}^{d=t_{h 2}-1} p_{d}$ and $\eta_{2}(T h)=\sum_{d=t_{h 2}-1}^{d=L-1} p_{d}$

The mean intensity distribution for $B_{0}, B_{1}$ and $B_{2}$ can be expressed as;

$$
\lambda_{0}=\sum_{d=0}^{t_{h 1}-1} \frac{d p_{d}}{\eta_{0}(T h)}, \lambda_{1}=\sum_{d=t_{h 1}}^{t_{h 2}-1} \frac{d p_{d}}{\eta_{1}(T h)} \text { and } \lambda_{2}=\sum_{d=t_{h 2}}^{t_{h 3}-1} \frac{d p_{d}}{\eta_{2}(T h)}
$$

The mean intensity of the whole image can be defined as; $\lambda_{T h}=\eta_{0} \lambda_{0}+\eta_{1} \lambda_{1}+\eta_{2} \lambda_{2}$, and $\eta_{0}+\eta_{1}+\eta_{2}=1$

Let the objective value to be maximized is;

$F_{O B C V_{\max }}(T h)=\mathrm{O}_{0}+\mathrm{O}_{1}+\mathrm{O}_{2}$

where $O_{0}=\eta_{0}\left(\lambda_{0}-\lambda_{T h}\right)^{2}, O_{1}=\eta_{1}\left(\lambda_{1}-\lambda_{T h}\right)^{2}$, and $O_{2}=\eta_{0}\left(\lambda_{2}-\lambda_{T h}\right)^{2}$

\subsection{Implementation}

In this work, the role of the proposed CCSA is to enhance the image for $T h=3$ using Kapur/Otsu. When the KE is employed, the objective function will be; $F_{\max }(X)=F_{K E_{\max }}(T h)$ and for Otsu's, this will be $F_{\max }(X)=F_{O B C V_{\max }}(T h)$. In the proposed work, the CCSA is initiated with the following values; no. of 
nests $=40$, search dimension $=T h=3$, Iter $_{\max }=3000$ and stopping function $=F_{\max }(X)$ or Iter $\max$. In this work, the experimental investigation is implemented using the proposed HIPS with the combinations, such as Kapur+LS, Kapur+CV, Otsu+LS and Otsu+CV and the corresponding results are recorded and analyzed.

\subsection{Image segmentation}

Segmentation of ROI from the enhanced image is widely employed by the researchers with automated and semi-automated techniques [17]. This work implemented the Level-Set (LS) as well as Chan-Vese (CV) segmentation procedure to extract the ROI from the thresholded CTS. The considered methods are semi-automated procedures works based on an initiated bounding-box. When the iteration increases, this box will converge towards the ROI and after identifying all the possible pixels of the ROI, the convergence of the box stops and presents the extracted COVID-19 infection. The essential information on the LS and the CV methods can be found in [17, 33-35].

\subsection{Performance computation and Validation}

The advantage of the proposed system is confirmed based on the performance measures computed using a comparative analysis between the ROI and GTI [36,37]. The performance measures commonly considered in medical image evaluation procedures are depicted below;

$$
\begin{aligned}
& \text { True Positive Rate }(T P R)=\text { Sensitivity }(\text { SEN })=\frac{T_{P}}{T_{P}+F_{N}} \\
& \text { True Negative Rate }(T N R)=\text { Specificity }(S P E)=\frac{T_{N}}{T_{N}+F_{P}} \\
& \text { False Negative Rate }(F N R)=\frac{F_{N}}{F_{N}+T_{P}}
\end{aligned}
$$$$
\text { False Positive Rate }(F P R)=\frac{F_{P}}{F_{P}+T_{N}}
$$$$
\operatorname{Accuracy}(A C C)=\frac{T_{P}+T_{N}}{T_{P}+T_{N}+F_{P}+F_{N}}
$$$$
\text { Precision }(P R E)=\frac{T_{P}}{T_{P}+F_{P}}
$$

Negative Predictive Value $(N P V)=\frac{T_{N}}{T_{N}+F_{N}}$

Jaccard Index $(J I)=\frac{I_{G} \cap I_{R}}{I_{G} \cup I_{R}}$

$$
\text { Dice }=\frac{2\left(I_{G} \cap I_{R}\right)}{\left|I_{G}\right| \cup\left|I_{R}\right|}
$$

where $T_{P}=$ true positive, $T_{N}=$ true negative, $F_{P}$ false positive, $F_{N}=$ false negative, $I_{G}=\mathrm{GTI}$, and $I_{R}=$ ROI 


\subsection{COVID-19 database}

The COVID-19 is a novel disease and the availability of the CTS dataset is very limited. The proposed work considers the lung CTS dataset of Jun et al. [21] for the assessment. This dataset consist 20 patient's information in 3D form. In the proposed research, 2D CTS are considered for the assessment and the sample test images collected from a patient along with the GTI is depicted in Figure 2.
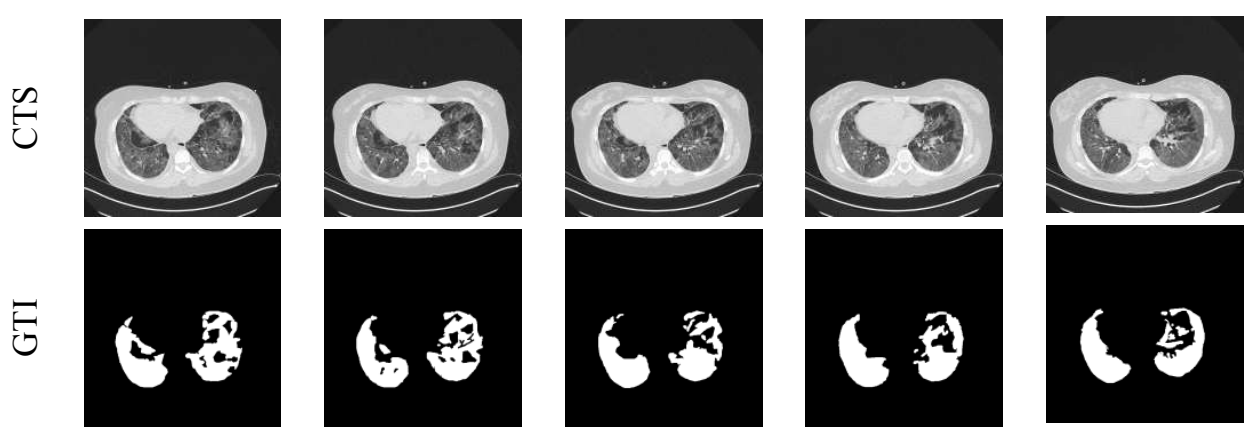

Figure 2: Sample test images and the corresponding GTI

\section{Results and Discussions}

This section of the research presents the results and discussions. Initially, the essential CTS are collected from the benchmark database provided by Jun et al. [21] and this dataset consist the test image along with the GTI. All the existing CTS are associated with the SOI and the unnecessary body organs. Hence, a threshold-filter discussed in [11] is employed to separate the considered test image. The threshold $(\mathrm{T})$ value for this filter is arbitrarily assigned $(\mathrm{T}=178)$ and the assigned value separates the image into two sections according to the threshold value (image section $1<\mathrm{T}<$ image section 2 ). From the separated image, the SOI is then considered for the assessment.

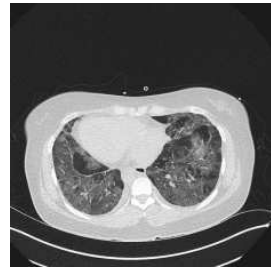

(a)

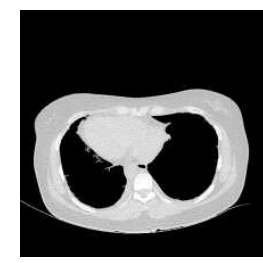

(b)

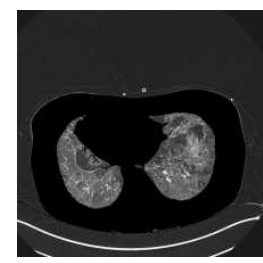

(c)

Figure 3: Results attained with threshold-filter for $\mathrm{T}=138$

(a) Test image, (b) Artifact, (c) SOI

The results attained by this filter are depicted in Figure 3. Fig 3(a) shows the test CTS and Fig (b) and (c) presents the outcome of the filter. Fig 3(c) is associated with the COVID-19 infection and hence, it is chosen as the SOI. The SOI image is then processed using the HIPS proposed in this research. Initially, the Kapur's approach based processing is implemented and then the considered image dataset is evaluated using the Otsu.

Figure 4 depicts the results attained with the HIPS implemented in this research. Fig 4 (a) to (d) presents the outcome attained with the Kapur's function and Fig 4(e) to (h) shows the results by Otsu. Initially, a CCSA and Kapur/Otsu based tri-level threshold is implemented to group the pixels of the SOI into three clusters, such as background, normal part and COVID-19. After enhancing the image, the COVID-19 infection is then extracted using the segmentation procedures, such as LS and CV. Figure 4 depicts the results of thresholding and LS segmentation. The outcome of this process depicted in Fig 4(d) and (h) is considered as the ROI and it is then compared against the GTI to judge the performance of the proposed approach. 


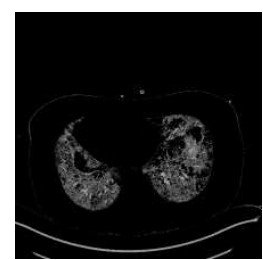

(a)

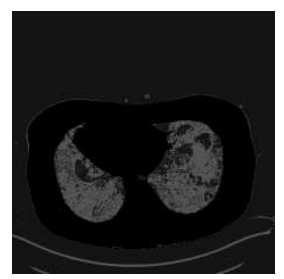

(e)

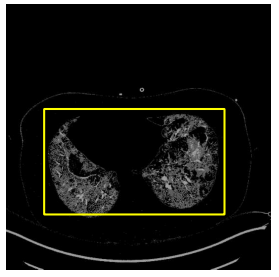

(b)

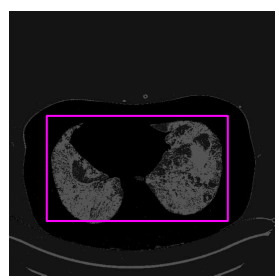

(f)

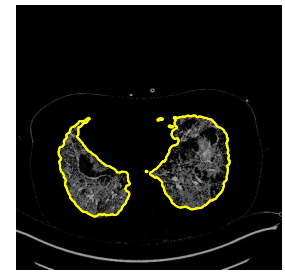

(c)

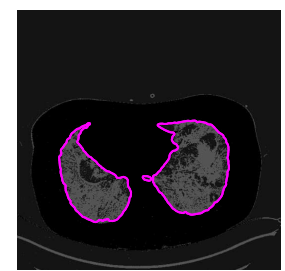

(g)

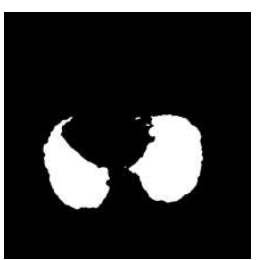

(d)

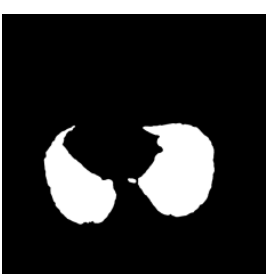

(h)

Figure 4: Results attained with the proposed image processing scheme

(a) Thresholded image with KE, (b) and (c) Initial and converged curve of LS, (d) Extracted ROI

(e) Thresholded image with Otsu, (f) and (g) Initial and converged curve, (d) Extracted COVID-19 infection

The ROI extraction from Fig 4(a) and (e) is then executed with the CV and the result attained for Fig 4(a) and 4(e) is depicted in Figure 5(a) and (c) respectively. Fig 5(b) and (d) depicts the extracted ROI for the Kapur+CV and Kapur+Otsu respectively.
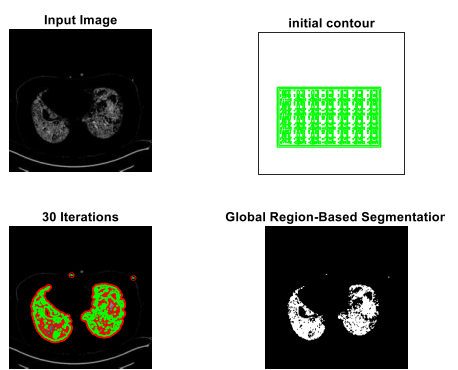

(a)
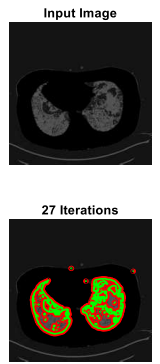
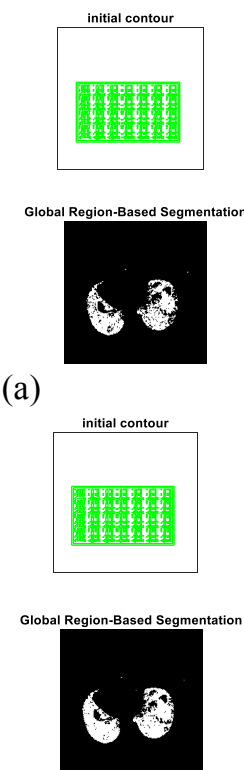

(c)

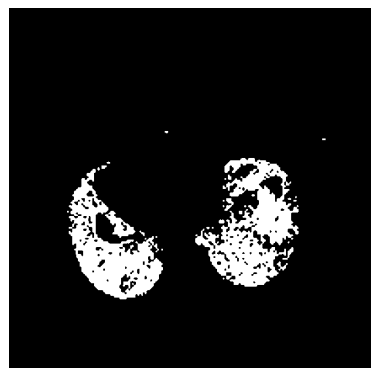

(b)

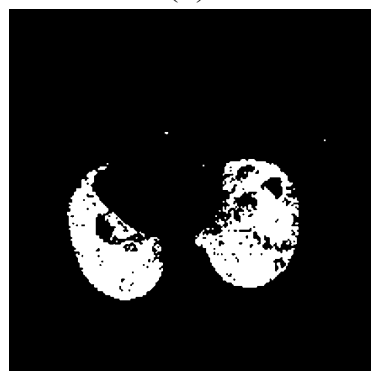

(d)

Figure 5. Results obtained with CV segmentation

(a) \& (b) Results attained with Kapur thresholding

(c) \& (d) Results attained with Otsu thresholding 
Table 1: Results attained during the pixel level comparison of the ROI with GTI

\begin{tabular}{ccccccc}
\hline Method & $\mathrm{T}_{\mathrm{P}}$ & $\mathrm{F}_{\mathrm{P}}$ & $\mathrm{T}_{\mathrm{N}}$ & $\mathrm{F}_{\mathrm{N}}$ & $\mathrm{FNR}$ & $\mathrm{FPR}$ \\
\hline Kapur+LS & 17432 & 569 & 346088 & 3682 & 0.1744 & 0.0016 \\
Otsu+LS & 17587 & 414 & 344969 & 3819 & 0.1784 & 0.0012 \\
Kapur+CV & 15171 & 2830 & 352738 & 1497 & 0.0898 & 0.0080 \\
Otsu+CV & 15462 & 1563 & 353552 & 984 & 0.0598 & 0.0044 \\
\hline
\end{tabular}

Table 2: Essential perormance measures computed with ROI and GTI comparison

\begin{tabular}{ccccccc}
\hline Method & SEN (TPR) & SPE (TNR) & ACC & PRE & JI & DICE \\
\hline Kapur+LS & 0.8256 & 0.9984 & 0.9884 & 0.9684 & 0.8039 & 0.8913 \\
Otsu+LS & 0.8216 & 0.9988 & 0.9885 & 0.9770 & 0.8060 & 0.8926 \\
Kapur+CV & 0.9102 & 0.9920 & 0.9884 & 0.8428 & 0.7781 & 0.8752 \\
Otsu+CV & 0.9402 & 0.9956 & 0.9931 & 0.9082 & 0.8586 & 0.9239 \\
\hline
\end{tabular}

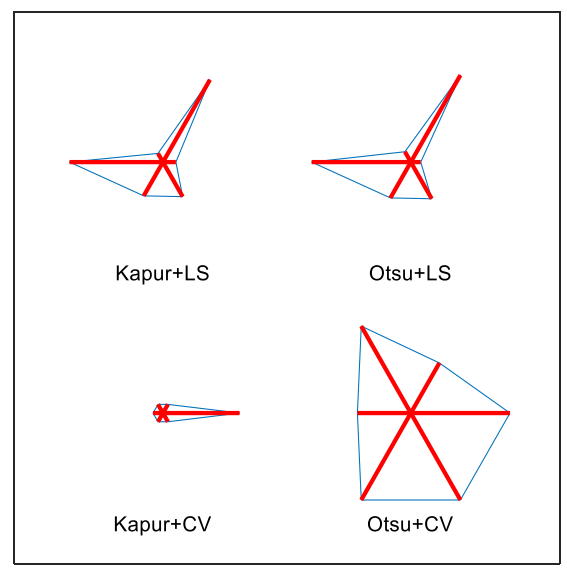

Figure 6: Glyph-plot for the performance measures presented in Table 2

Table 1 and 2 presents the performance measures attained with a relative assessment between the extorted ROI and GTI for the Kapur/Otsu thresholding and LS/CV segmentation cases. The individual assessment of the attained performance for a single image case is quite confusing and hence a graphical 
depiction is always preferred for the assessment. In order to identify the best approach, a glyph-plot [38] is constructed as in Figure 6. The glyph-plot helps to identify the overall performance of the values based on its relative analysis. The larger dimension of the plot confirms the superiority of the variables considered for the assessment. In this work, the essential plot is created using the Table 2 values and from this plot it can be observed that, the Otsu+CV based HIPS offers superior results (larger dimension) compared with the alternatives. Similar procedure is executed on all the 100 test images and the mean value of the segmentation accuracy is considered for the assessment as shown in Figure 7. From this figure, the HIPS based on Otsu+CV helped to attain better segmentation accuracy compared to Kapur+LS, Otsu+LS and Kapur+CV.

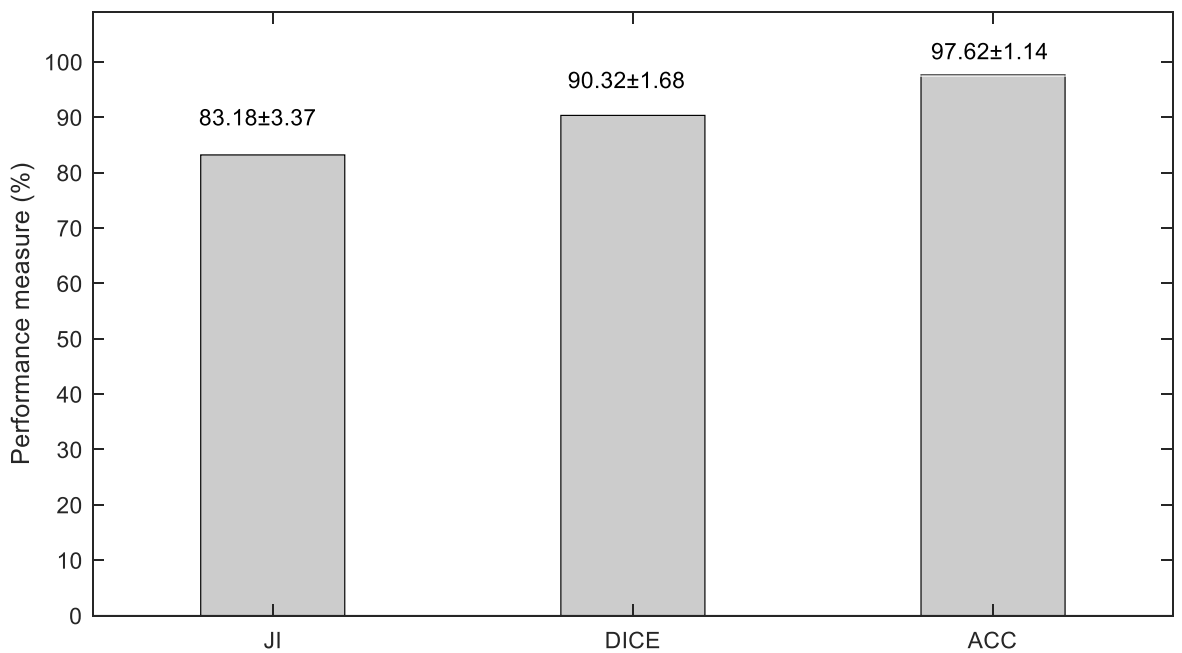

Figure 7: Validation of the performance of the proposed procedure based on the segmentation accuracy

This work presented an image processing based assessment of the COVID-19 infection using the 2D CTS. This work is implemented only for the COVID-19 infection segmentation task using a chosen CTS database and in future, the performance of this procedure can be implemented on the clinical grade CTS images collected from real patients. Further, a chosen machine-learning and deep-learning procedure can be implemented to support the automated detection of the COVID-19 infection using the CTS image dataset.

\section{Conclusion}

Due to its medical emergency, COVID-19 lesion assessment emerged as an important research domain and a considerable number of modelling and image examination techniques are proposed and implemented with clinical level and open source datasets. The proposed work implemented a hybrid image evaluation scheme to examine the COVID-19 infection using the lung CTS. A combination of a chosen thresholding and segmentation procedure is implemented to extract the ROI for the analysis. In this work, 100 number of test images of dimension 630x630x1 pixels with the COVID-19 infection are considered for the experimental investigation. A tri-level threshold with Chaotic CSA and Kapur/Otsu is initially executed to enhance the COVID-19 lesion in test image and then the enhanced sections are segmentation using LS/CV technique, in order to extract the ROI. After extraction the essential ROI (COVID-19 lesion), a relative study is implemented between the ROI and GTI and the essential performance measures are computed. The result attained with the proposed experimental study confirms that, the proposed system with Otsu $+\mathrm{CV}$ offers better mean values of JI (83.18 \pm 3.37$)$, Dice (90.32 \pm 1.68$)$ and segmentation accuracy $(97.62 \pm 1.14)$ compared to Kapur+LS, Otsu+LS and Kapur+CV. This result confirms that, proposed image evaluation system works well on the considered image dataset. The 
attained result of this study confirms its clinical significance and in future, it can be considered to examine the real clinical CT scan slices with COVID-19 lesion collected from hospitals.

Funding Statement: The author(s) received no specific funding for this study.

Conflicts of Interest: The authors declare that they have no conflicts of interest to report regarding the present study.

\section{References}

1. https://www.who.int/news-room/fact-sheets/detail/pneumonia (Last accessed date 1st May 2020)

2. Asnaoui, K.E.; Chawki, Y.; Idri, A. (2020): Automated Methods for Detection and Classification Pneumonia based on X-Ray Images Using Deep Learning. arXiv:2003.14363.

3. Bhandary, A. et al. (2020): Deep-learning framework to detect lung abnormality-A study with chest X-Ray and lung CT scan images. Pattern Recognition Letters, vol.129, pp.271-278. https://doi.org/10.1016/j.patrec.2019.11.013.

4. Rajpurkar, P. et al. (2017): CheXNet: radiologist-level pneumonia detection on chest x-rays with deep learning, arXiv preprint. arXiv:1711.05225 [cs.CV].

5. Syrjala, H. et al. (2017): Chest magnetic resonance imaging for pneumonia diagnosis in outpatients with lower respiratory tract infection. European Respiratory Journal, vol.49, pp.1601303. DOI: $10.1183 / 13993003.01303-2016$

6. Zech, J.R. et al. (2018): Variable generalization performance of a deep learning model to detect pneumonia in chest radiographs: a cross-sectional study. PLoS Med., vol.15, no.11, pp. e1002683.

7. https://www.worldometers.info/coronavirus/ (Last accessed date 21st May 2020)

8. https://www.nejm.org/coronavirus (Last accessed date 21st May 2020

9. https://www.who.int/emergencies/diseases/novel-coronavirus-2019 (Last accessed date 21st May 2020)

10. https://www.cdc.gov/coronavirus/2019-ncov/index.html (Last accessed date 21st May 2020)

11. Rajinikanth, V.; Dey, N.; Raj, A.N.J.; Hassanien, A.E.; Santosh, K.C.; Raja, N.S.M. (2020): Harmonysearch and Otsu based system for coronavirus disease (COVID-19) detection using lung CT scan images, arXiv preprint. arXiv:2004.03431.

12. Rajinikanth, V.; Kadry, S.; Thanaraj, K.P.; Kamalanand, K.; Seo, S. (2020): Firefly-Algorithm Supported Scheme to Detect COVID-19 Lesion in Lung CT Scan Images using Shannon Entropy and MarkovRandom-Field, arXiv preprint. arXiv:2004.09239.

13. Kadry, S.; Rajinikanth, V.; Rho, S.; Raja, N.S.M.; Rao, V.S.; Thanaraj, K.P. (2020); Development of a Machine-Learning System to Classify Lung CT Scan Images into Normal/COVID-19 Class, arXiv preprint. arXiv:2004.13122.

14. Dey, N.; Rajinikant, V.; Fong, S.J.; Kaiser, M.S.; Mahmud, M. (2020): Social-Group-Optimization Assisted Kapur's Entropy and Morphological Segmentation for Automated Detection of COVID-19 Infection from Computed Tomography Images. Preprints, 2020050052. DOI: 10.20944/preprints202005.0052.v1.

15. Wu, Y-H.; Gao, S-H.; Mei, J.; Xu, J.; Fan, D-P.; Zhao, C-W.; Cheng, M-M. (2020): JCS: An Explainable COVID-19 Diagnosis System by Joint Classification and Segmentation, arXiv preprint. arXiv:2004.07054.

16. Fan, D-P.; Zhou, T.; Ji, G-P.; Zhou, Y.; Chen, G.; Fu, H.; Shen, J.; Shao, L. (2020): Inf-Net: Automatic COVID-19 Lung Infection Segmentation from CT Scans, arXiv preprint. arXiv:2004.14133

17. Rajinikanth, V. et al. (2017): Entropy based segmentation of tumor from brain MR images-a study with teaching learning based optimization, Pattern Recognition Letters, vol.94, pp.87-95. https://doi.org/10.1016/j.patrec.2017.05.028.

18. Khan, M.A. et al. (2020). Gastrointestinal diseases segmentation and classification based on duo-deep architectures, Pattern Recognition Letters, vol.131, pp.193-204. https://doi.org/10.1016/j.patrec.2019.12.024. 
19. Asokan, A. et al. (2020): Bat algorithm based non-linear contrast stretching for satellite image enhancement, Geosciences, vol.10, no.2, pp.78. https://doi.org/10.3390/geosciences10020078.

20. Hemanth, D.J.; Deperlioglu, O.; Kose, U. (2020). An enhanced diabetic retinopathy detection and classification approach using deep convolutional neural network, Neural Computing and Applications, vol.32, no.2, pp.707-721. https://doi.org/10.1007/s00521-018-03974-0.

21. Jun, M.; Cheng, G.; Yixin, W.; Xingle, A.; Jiantao, G.; Yu Ziqi, ... He Jian. (2020). COVID-19 CT Lung and Infection Segmentation Dataset (Version Verson 1.0) [Data set]. Zenodo. http://doi.org/10.5281/zenodo.3757476.

22. Stewart, I. (2000): The Lorenz Attractor Exists. Nature vol.406, pp.948-949.

23. Yüzgeç, U.; Eser, M. (2018): Chaotic based differential evolution algorithm for optimization of baker's yeast drying process. Egyptian Informatics Journal, vol.19, no.3, pp.151-163. https://doi.org/10.1016/j.eij.2018.02.001.

24. Yang, X.S.; Deb, S. (2009): Cuckoo search via Lévy flights. In: Proceedings of World Congress on Nature and Biologically Inspired Computing (NaBIC 2009), IEEE Publications, USA, pp. 210-214. DOI: 10.1109/NABIC.2009.5393690 .

25. Yang, X.S. (2008): Nature-Inspired Metaheuristic Algorithms, Luniver Press, Frome, UK.

26. Brajevic, I., Tuba, M. (2014): Cuckoo search and firefly algorithm applied to multilevel image thresholding. In: Cuckoo Search and Firefly Algorithm. Studies in Computational Intelligence, vol. 516, pp. 115-139. Springer, Heidelberg.

27. Bhandari, A.K.; Singh, V.K.; Kumar, A.; Singh, G.K. (2014): Cuckoo search algorithm and wind driven optimization based study of satellite image segmentation for multilevel thresholding using Kapur's entropy. Expert Syst. Appl. vol.41, no.7, pp.3538-3560.

28. Agrawala, S.; Pandaa, R.; Bhuyana, S.; Panigrahi, B.K. (2013): Tsallis entropy based optimal multilevel thresholding using cuckoo search algorithm. Swarm Evol. Comput. vol.11, pp.16-30.

29. Kapur, J.N.; Sahoo, P.K.; Wong, A.K.C. (1985): A new method for gray-level picture thresholding using the entropy of the histogram. Comput. Vis. Graph. Image Process. vol.29, pp.273-285.

30. Bhandari, A.K.; Kumar, A.; Singh, G.K. (2015): Modified artificial bee colony based computationally efficient multilevel thresholding for satellite image segmentation using Kapur's, Otsu and Tsallis functions. Expert Syst. Appl. vol.42, pp.1573-1601.

31. Otsu, N. (1979): A threshold selection method from gray-level histograms. IEEE Trans Syst Man Cybern., vol.9, no.1, pp.62-66.

32. Satapathy, S.C.; Raja, N.S.M.; Rajinikanth, V. et al. (2018): Multi-level image thresholding using Otsu and chaotic bat algorithm. Neural Comput \& Applic., vol. 29, pp.1285-1307. https://doi.org/10.1007/s00521-0162645-5.

33. Li, C.; Xu, C.; Gui, C.; Fox, M.D. (2010): Distance regularized level set evolution and its application to image segmentation, IEEE Transactions on Image Processing, vol.19, no.12, pp. 3243-3254.

34. Chan, T.F.; Vese, L.A. (2001): Active contours without edges. IEEE Trans Image Process., vol.10, no.2, pp.266-277.

35. Dey, N. et al. (2019): Leukocyte nuclei segmentation using entropy function and Chan-Vese approach, Information Technology and Intelligent Transportation Systems, vol.314, pp.255-264.

36. Dey, N. et al. (2019): Social-Group-Optimization based tumor evaluation tool for clinical brain MRI of Flair/diffusion-weighted modality. Biocybernetics and Biomedical Engineering, vol.39, no.3, pp.843-856. https://doi.org/10.1016/j.bbe.2019.07.005

37. Acharya, U.R. et al. (2019): Automated detection of Alzheimer's disease using brain MRI images-a study with various feature extraction techniques. Journal of Medical Systems, vol.43, no.9, pp.302. https://doi.org/10.1007/s10916-019-1428-9

38. Ward, M.O. (2008): Multivariate Data Glyphs: Principles and Practice. Handbook of Data Visualization, pp.179-198. https://doi.org/10.1007/978-3-540-33037-0_8.

39. http://www.itksnap.org/pmwiki/pmwiki.php 
40. Yushkevich, P.A.; Piven, J.; Hazlett, H.C.; Smith, R.G.; Ho, S.; Gee, J.C.; Gerig, G. (2006): User-guided 3D active contour segmentation of anatomical structures: significantly improved efficiency and reliability. Neuroimage, vol.31, no.3, pp.1116-1128.

41. Ozturk, T.; Talo, M.; Yildirim, E.A.; Baloglu, U.B.; Yildirim, O.; Acharya, U.R. (2020): Automated detection of COVID-19 cases using deep neural networks with X-ray images, Computers in Biology and Medicine, vol.121, pp. 103792.

42. Alazab, M.; Awajan, A.; Mesleh, A.; Abraham, A.; Jatana, V.; Alhyari, S. (2020): COVID-19 Prediction and Detection Using Deep Learning, International Journal of Computer Information Systems and Industrial Management Applications, vol. 12, pp. 168-181.

43. Singh, D., Kumar, V., Vaishali et al. (2020). Classification of COVID-19 patients from chest CT images using multi-objective differential evolution-based convolutional neural networks. European Journal of Clinical Microbiology \& Infectious Diseases. https://doi.org/10.1007/s10096-020-03901-z.

44. Ardakani, A.A.; Kanafi, A.R.; Acharya, U.R.; Khadem, N.; Mohammadi, A. (2020): Application of deep learning technique to manage COVID-19 in routine clinical practice using CT images: Results of 10 convolutional neural networks, Computers in Biology and Medicine, vol.121, pp.103795.

45. Ahuja, S.; Panigrahi, B.K.; Dey, N.; Gandhi, T.K.; Rajinikanth, V. (2020). Deep transfer learning-based automated detection of COVID-19 from lung CT scan slices. TechRxiv. 


\section{Figures}

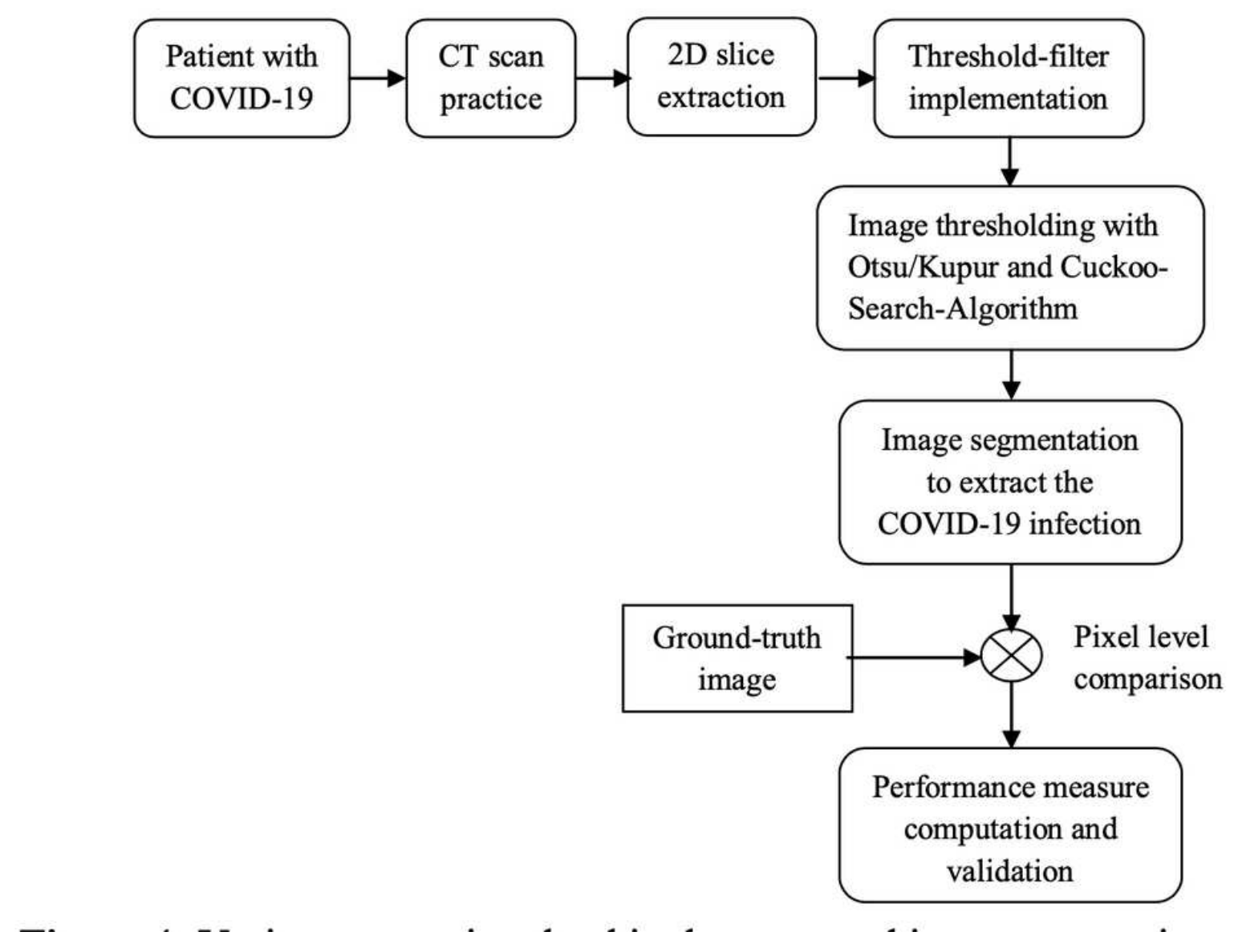

Figure 1

Various stages involved in the proposed image processing scheme
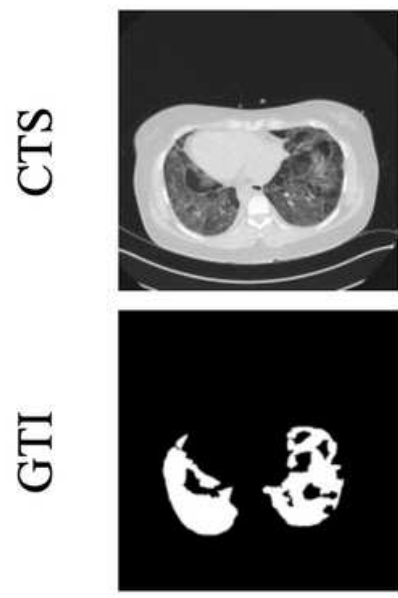
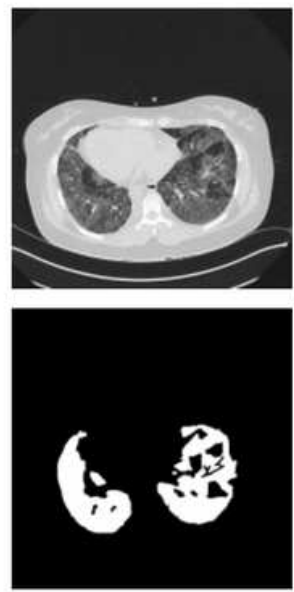
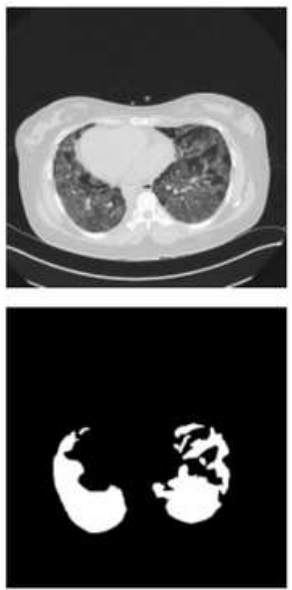
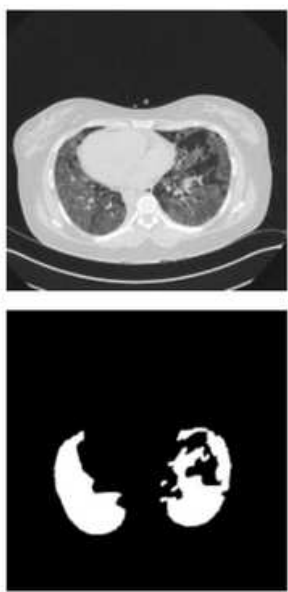
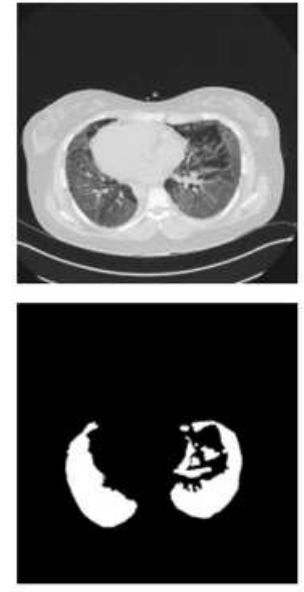

Figure 2 


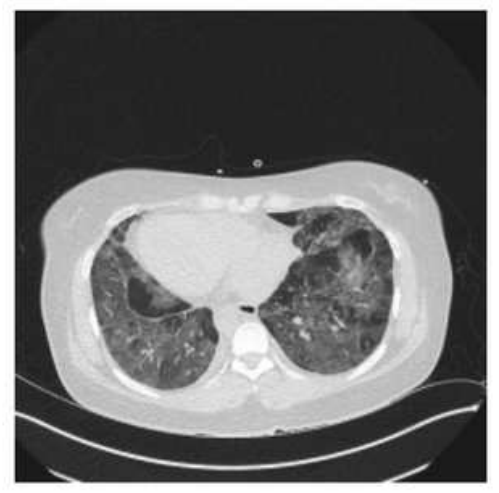

(a)

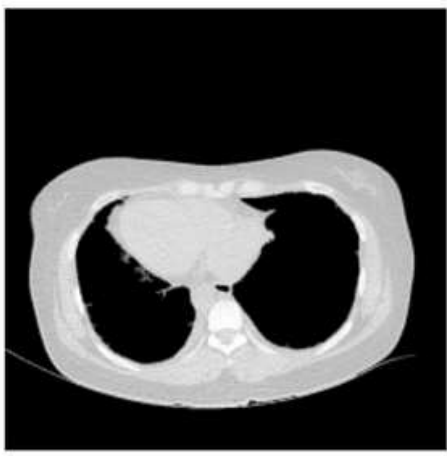

(b)

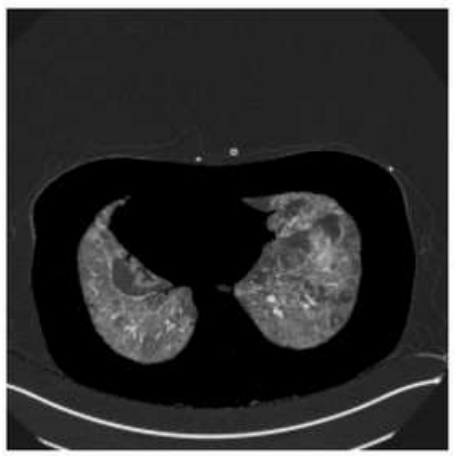

(c)

\section{Figure 3}

Results attained with threshold-filter for T=138 (a)Test image, (b) Artifact, (c) SOI

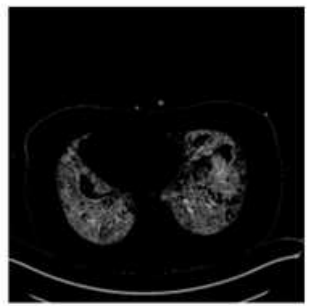

(a)

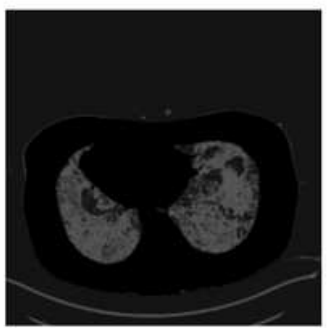

(e)

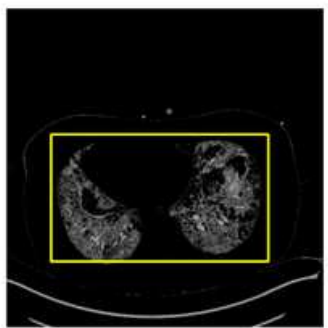

(b)

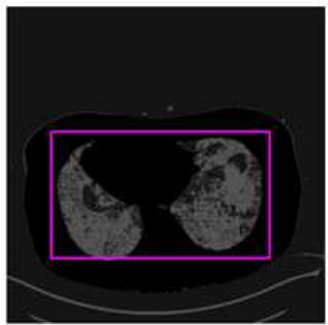

(f)

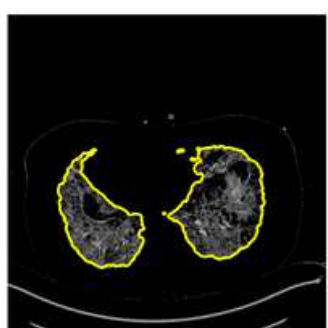

(c)

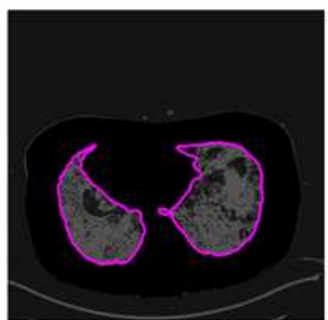

(g)

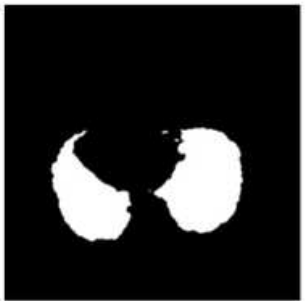

(d)

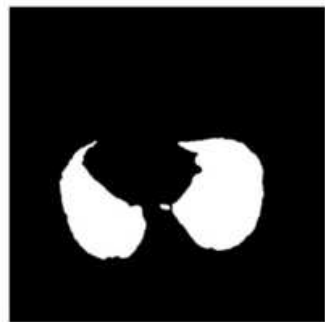

(h)

\section{Figure 4}

Results attained with the proposed image processing scheme (a) Thresholded image with KE, (b) and (c) Initial and converged curve of LS, (d) Extracted ROI (e) Thresholded image with Otsu, (f) and (g) Initial and converged curve, (h) Extracted COVID-19 infection 
Input Image

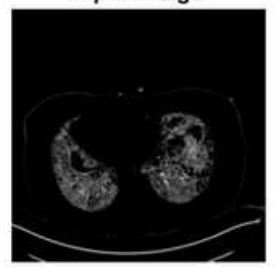

30 Iterations

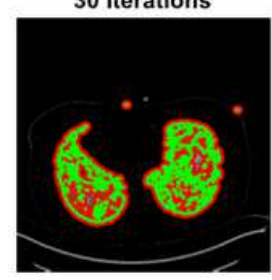

Input Image

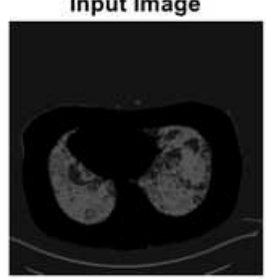

27 Iterations

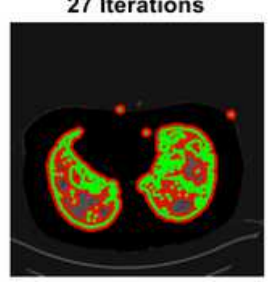

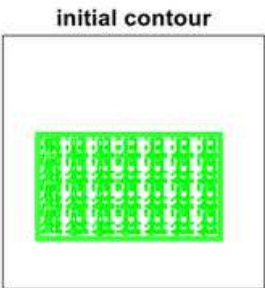

Global Region-Based Segmentatior

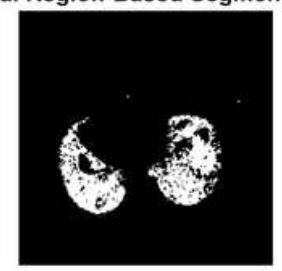

(a)

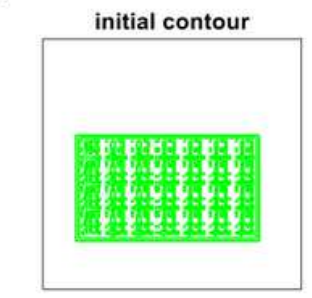

Global Region-Based Segmentation

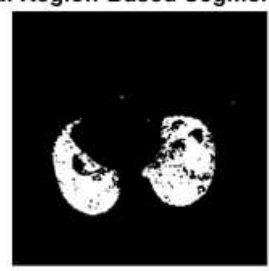

(c)

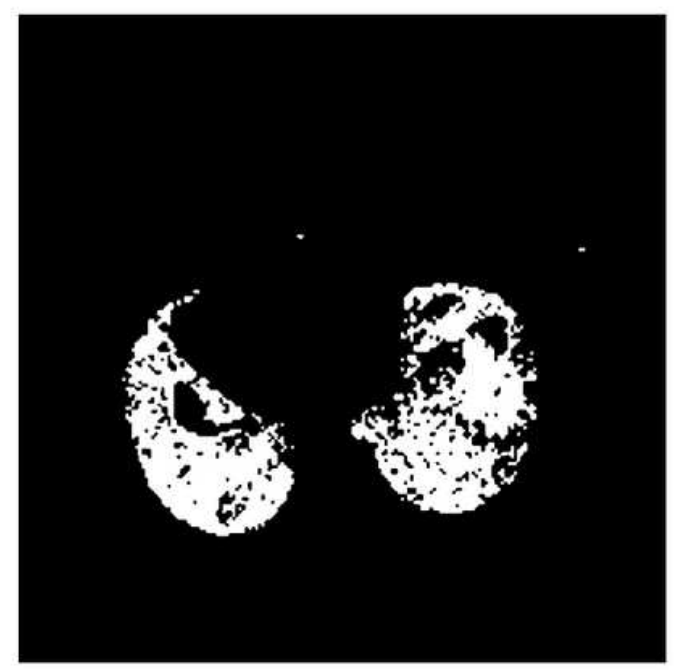

(b)

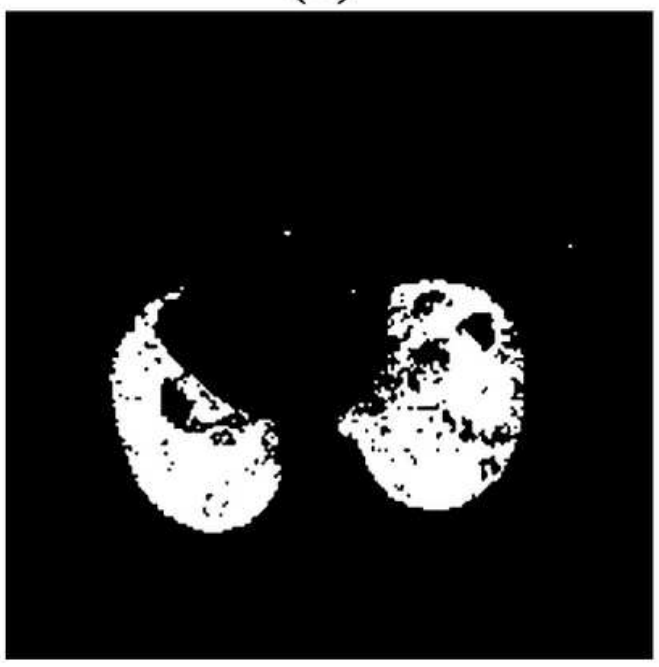

(d)

\section{Figure 5}

Results obtained with CV segmentation (a) \& (b) Results attained with Kapur thresholding (c) \& (d) Results attained with Otsu thresholding 


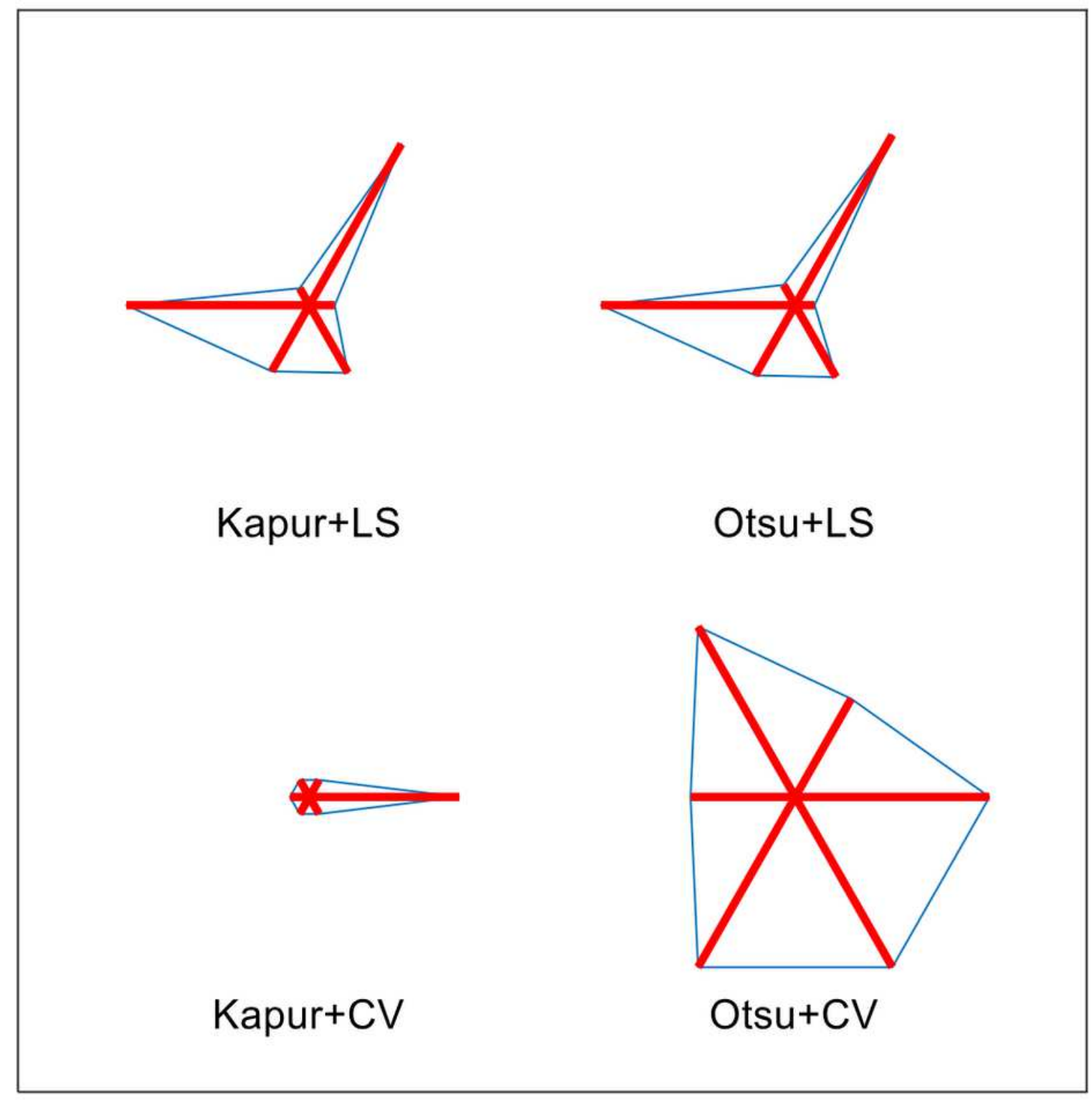

Figure 6

Glyph-plot for the performance measures presented in Table 2 


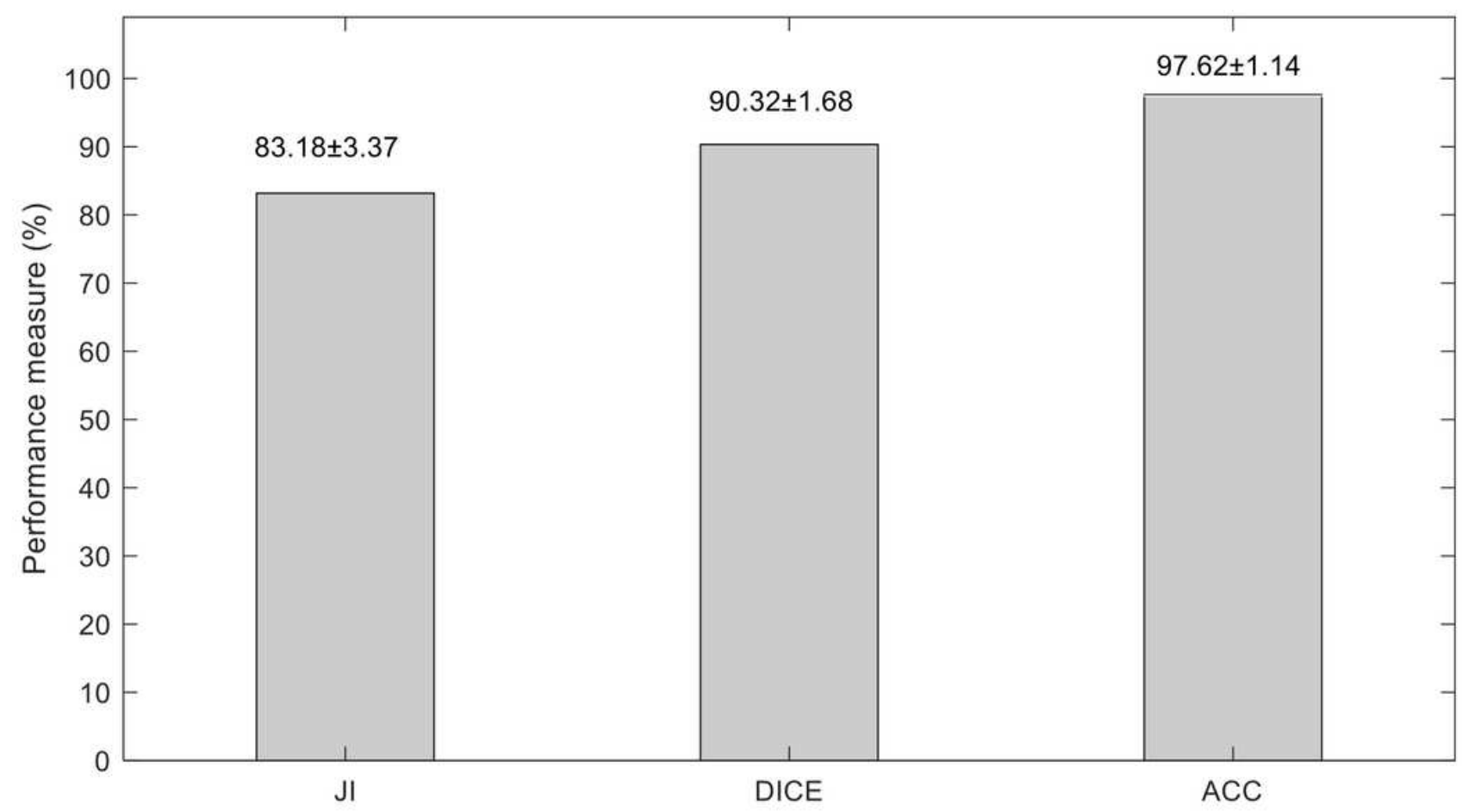

Figure 7

Validation of the performance of the proposed procedure based on the segmentation accuracy 